\title{
Percepção dos idosos jovens e longevos gaúchos quanto aos espaços públicos em que vivem
}

\author{
The perception of the young and long-lived elderly 'Gauchos' \\ (from the State of Rio Grande do Sul, Brazil) \\ about the public spaces they live in
}

Joel Hirtz do Nascimento Navarro ${ }^{1}$

Francini Porcher Andrade ${ }^{2}$

Tiago Sousa Paiva ${ }^{3}$

Diovana Ourique da Silva ${ }^{2}$

Cristiane Fernanda Gessinger ${ }^{2}$

Ângelo José Gonçalves Bós ${ }^{1}$

${ }^{1}$ Instituto de Geriatria e Gerontologia, Pontifícia Universidade Católica do Rio Grande do Sul. Av. Ipiranga 6681, Partenon 90619-900 Porto Alegre RS Brasil. joelhnn@hotmail. com

${ }^{2}$ Instituto Porto Alegre, Centro Universitário Metodista.

${ }^{3}$ Programa de Valorização do Profissional da Atenção Básica, Ministério da Saúde.
Abstract By 2050, the number of Brazilians living in urban areas will be over 200 million and $29 \%$ of the population will be elderly. The longlived elderly are 80 or more years old and the young elderly are between 60 and 79 years of age. The scope of this article was to verify the difference in perception between the young elderly and the long-lived elderly from Rio Grande do Sul (RS) about the urban environment they live in. This is a population-based, observational, descriptive, retrospective study with a quantitative analysis paradigm. Data was analyzed from Elderly Profile research in $R S$ conducted by the Geriatric and Gerontological Institute of PUCRS in partnership with the RS School of Public Health. The sample consisted of 6913 questionnaires answered by the elderly from 59 cities. Data analysis was performed for each age group and independent variables were processed using the Chi-square test, with $p$ under 0.05. Results showed that the perception of difficulties such as a lack of park benches and safety strips, short traffic light times for pedestrians, high steps and bad-smelling public toilets was greater among the young elderly. The long-lived elderly noticed these facts less, though they admitted that they frequent community environments less often.

Key words Long-lived elderly, Active aging, Urban mobility, City planning
Resumo Em 2050, o número de brasileiros residindo em áreas urbanas passará dos 200 milhões e 29\% da população será composta por idosos. Os idosos longevos possuem 80 anos ou mais, os idosos jovens são aqueles que apresentam idade entre 60 e 79 anos. O objetivo foi verificar a diferença da percepção de idosos jovens e longevos do Rio Grande do Sul quanto ao ambiente urbano em que vivem. Estudo de base populacional, observacional, descritivo, retrospectivo, com paradigma de análise quantitativa, analisou dados da pesquisa Perfil dos Idosos do RS, realizado pelo Instituto de Geriatria e Gerontologia da PUCRS em parceria com a Escola de Saúde Pública do RS. A amostra foi composta por 6913 questionários respondidos por idosos de 59 cidades. A análise dos dados foi realizada com cada um dos grupos etários e as variáveis independentes foram testadas pelo Qui-Quadrado, sendo o nível de significância menor que 0,05. Como resultado, a percepção de dificuldades, como poucos bancos, falta de faixas de segurança, tempo de sinal muito curto para pedestres, degraus muito altos e mau cheiro dos banheiros públicos, foi maior entre os idosos jovens. Os idosos longevos perceberam menos esses fatores, porém, referiram que frequentam menos os ambientes comunitários.

Palavras-chave Idoso longevo, Envelhecimento ativo, Mobilidade urbana, Planejamento de cidades 


\section{Introdução}

O crescimento do contingente populacional que reside em espaços urbanos no Brasil é evidente. Essa tendência pode ser observada quando comparadas as estimativas de indivíduos habitantes de áreas urbanas e rurais para o ano de 2050 no país. A população nas grandes cidades está estimada para mais de 200 milhões de habitantes ${ }^{1}$. Enquanto a perspectiva de indivíduos residentes em áreas rurais se aproxima dos 20 milhões $^{2}$. Em paralelo às estimativas de urbanização da população brasileira tem-se uma grande perspectiva de envelhecimento populacional, no qual a projeção de pessoas com 60 anos ou mais para o mesmo ano corresponde a $29 \%$ da população total brasileira ${ }^{3}$.

Um país urbanizado e com crescentes taxas de segmentos populacionais mais velhos necessita de planejamentos em longo prazo para garantir uma sociedade para todos. Enfatizar aspectos de habitação, serviços de saúde, meio ambiente, educação e oportunidades para que as diversas faixas etárias possam estabelecer vínculos e integrar-se ao ambiente em que vivem torna-se essencial $^{4}$.

A construção de sociedades integradas para todos pode ter início a partir das Cidades Amigas do Idoso. A Organização Mundial da Saúde (OMS) define como cidade amiga do idoso aquela que estimula o envelhecimento ativo ao otimizar as oportunidades para a saúde, a participação e a segurança, com o objetivo de aumentar a qualidade de vida no envelhecimento. Modifica e adapta suas estruturas de forma que a população idosa possa interagir com o ambiente comunitário nas suas diferentes realidades ${ }^{5}$.

Aspectos do meio urbano são determinantes de um envelhecimento ativo e saudável, tais como a qualidade dos espaços abertos e prédios, o apoio comunitário e serviços de saúde, a comunicação e a informação, a participação cívica e o emprego, o respeito e a inclusão social, a participação social, a moradia e o transporte ${ }^{6}$.

A percepção que os idosos possuem do meio urbano é determinante de suas relações com a cidade, um idoso que sente-se inseguro para sair às ruas, sem oportunidades de participação e privado de mobilização por falta de uma estrutura urbana adequada, acaba enclausurado em sua residência. Já um ambiente sem obstáculos, que oferece condições confortáveis para movimentação proporciona maior contato do idoso com a cidade ${ }^{7}$.

Os idosos de diversas faixas etárias podem reagir diferentemente frente aos obstáculos do ambiente, para melhor captar essa diferença surge a subdivisão dos idosos em longevos e jovens. Os longevos são aqueles que possuem 80 anos ou mais de idade, sendo considerados idosos jovens aqueles que se caracterizam como não longevos $(60 \text { a } 79 \text { anos })^{8,9}$. O Rio Grande do Sul (RS) é o quarto estado brasileiro com maior número de idosos segundo o CENSO de $2010^{10}$, torna-se notória a necessidade de garantir que os idosos jovens e longevos possam desfrutar de todos os bens que a sociedade possa lhes proporcionar.

O presente estudo tem como objetivo verificar a diferença da percepção que os idosos jovens e longevos do Rio Grande do Sul possuem do ambiente urbano em que vivem. Através da verificação dessa diferença de percepção, poderão ser identificadas as necessidades específicas de cada um, enfatizando os dados relacionados ao grupo mais velho, uma vez que é um segmento com poucas referências na literatura científica, carecendo de informações que possam qualificar as estratégias para o seu cuidado. Conhecendo a realidade desses idosos, podem-se evidenciar as características positivas ou negativas que as cidades do RS oferecem para a interação social desse contingente populacional, redimensionando ações relacionadas à promoção da saúde desta população.

\section{Métodos}

O presente estudo caracteriza-se por ser de base populacional, observacional, descritivo, retrospectivo, com paradigma de análise quantitativa. Analisou dados oriundos do banco de dados da pesquisa Perfil dos Idosos do RS, realizada pelo Instituto de Geriatria e Gerontologia da PUCRS (IGG-PUCRS) em parceria com a Escola de Saúde Pública do RS (ESP) nos anos de 2010 e 2011. O questionário aplicado aos idosos na pesquisa de base fundamentou-se no relatório da Organização Mundial da Saúde "Guia Global: cidade amiga do idoso". O instrumento final foi composto por 72 questões fechadas com escolha simples ou múltipla, agrupadas em 13 blocos temáticos sendo eles: dados gerais do idoso, características do idoso e seu ambiente, transporte, habitação, renda, saúde, respeito e inclusão social, participação social, cívica e laboral, sexualidade, comunicação e informação, suporte comunitário e serviços de saúde, composição familiar e ocupação.

A amostra estudada no presente estudo foi composta por 6913 questionários respondidos por idosos de 60 anos ou mais, de ambos os gêneros, residentes em áreas urbanas de 59 municí- 
pios do RS. Foram excluídos das análises os questionários dos idosos que residem em áreas rurais, o critério de exclusão foi pagar o imposto territorial rural (ITR). O comprometimento cognitivo também foi um critério de exclusão, identificado pela impossibilidade de memorizar duas de três palavras solicitadas.

Foram analisadas as respostas de 5 questões da pesquisa de base selecionadas a partir do seguinte tema: percepção dos idosos jovens e longevos quanto aos locais públicos urbanos que frequentam. A coleta de dados retrospectivos ocorreu de fevereiro à março de 2013. A análise dos dados foi realizada com cada um dos grupos etários: idosos jovens (60 a 79 anos) e idosos longevos (80 anos ou mais), para que assim a diferença de percepção dos mesmos quanto ao ambiente urbano pudesse ser verificada.

As tabelas de cruzamento das respostas dos idosos jovens e longevos referentes às variáveis independentes (categóricas) foram testadas pelo Qui-quadrado. Foram considerados significativos os testes que corresponderam a um nível de significância menor ou igual que 0,05 . Os dados coletados foram tabulados através do software Epi Info, versão 3,5.

O projeto de pesquisa que gerou o banco de dados foi aprovado pelo Comitê de Ética e Pesquisa da Escola de Saúde Pública e pelo Comitê de Ética em pesquisa da PUCRS. Nessa pesquisa todos os entrevistados assinaram o termo de consentimento livre e esclarecido. O banco de dados analisado não continha informações pessoais dos entrevistados, desta forma a análise foi feita respeitando o sigilo de suas identidades.

\section{Resultados}

Foram analisadas as respostas quanto à percepção do ambiente urbano a partir de um questionário respondido por 6913 indivíduos, dentre estes, 6056 idosos jovens e 857 idosos longevos, sendo $51,6 \%$ mulheres e $48,4 \%$ homens. Não houve diferença estatisticamente significativa na distribuição do gênero entre os idosos jovens e os longevos.

Quanto à frequência de sair de casa semanalmente, 6030 idosos no total de entrevistas analisadas responderam que saem de sua residência com essa frequência, sendo o percentual de idosos longevos que não saem de casa semanalmente significativamente maior do que no grupo de idosos jovens (Tabela 1).

A falta de segurança foi a maior dificuldade enfrentada para sair de casa, referida por 1378 entrevistados, tanto longevos como jovens, não havendo diferença estatística entre os grupos. A dificuldade de locomoção foi a segunda opção mais relatada, correspondendo a 166 idosos longevos e 424 idosos jovens, sendo significativamente mais frequente no grupo mais velho. $\mathrm{O}$ fato de não se sentirem motivados foi mais referido entre os idosos longevos demonstrando diferença estatisticamente significativa entre os grupos. A falta de ter com quem sair, a dificuldade de enxergar e a dificuldade com o transporte coletivo ou privado, obtiveram percentuais significativos e maiores entre os idosos longevos. As dificuldades encontradas pelos entrevistados foram percentualmente maiores entre o grupo mais velho, o que sugere uma maior limitação destes para saírem de casa (Tabela 2).

Sobre a percepção dos locais públicos frequentados, os ambientes inseguros foram relatados por 1590 idosos jovens e 215 longevos, somando $26,1 \%$ dos indivíduos entrevistados. Não houve diferença significativa entre os dois grupos, ambos percebem essa limitação do espaço público, conforme Tabela 3. A alternativa "poucos bancos ou em mal estado" foi significativamente mais relatada pelos idosos jovens (19,6\%). Os locais públicos "sujos, sem limpeza" foram evidenciados por 1024 pessoas, não obtendo diferença significativa entre as duas faixas etárias. Ambientes pouco iluminados foram significativamente mais percebidos pelos idosos jovens, correspondendo a 542 participantes do estudo. No total, 1508 entrevistados declararam não saber responder, pois não frequentam os locais pú-

Tabela 1. Frequência semanal que o idoso sai de casa.

\begin{tabular}{lcccc}
\hline & Idosos Jovens & Idosos Longevos & & \\
& $\mathbf{N}(\%)$ & $\mathbf{N}(\%)$ & Total & Valor $\mathbf{p}$ \\
\hline Sai de casa semanalmente & $5.379(90,8)$ & $651(77,5)$ & $6.030(89,1 \%)$ & $\mathrm{P}<0,001$ \\
Não sai de casa semanalmente & $545(9,2)$ & $189(22,5)$ & $734(10,9 \%)$ & \\
\hline
\end{tabular}


blicos, sendo este percentual significativamente maior no grupo mais velho (Tabela 3).

Quando questionados quanto às principais barreiras para atravessar as vias públicas, a maior dificuldade apontada foi a de que os carros não param na faixa de pedestre, relatada por 1181 idosos jovens e 149 longevos, não havendo diferença estatística entre os dois grupos (Tabela 4).

Tabela 2. Dificuldades encontradas para sair de casa.

\begin{tabular}{lrrrr}
\hline & Idosos Jovens & Idosos Longevos & & \\
& $\mathbf{N}(\%)$ & $\mathbf{N}(\%)$ & Total & Valor p \\
\hline Falta de segurança & $1.188(19,6)$ & $190(22,2)$ & $1.378(19,9 \%)$ & $\mathrm{p}=0,079$ \\
Dificuldade de locomoção & $424(7)$ & $166(19,4)$ & $590(8,5 \%)$ & $\mathrm{p}<0,001$ \\
Não se sente motivado (a) & $289(4,8)$ & $71(8,3)$ & $360(5,2 \%)$ & $\mathrm{p}<0,001$ \\
Falta de ter com quem sair & $180(3)$ & $46(5,4)$ & $226(3,3 \%)$ & $\mathrm{p}<0,001$ \\
Necessidade de banheiro com frequência & $121(2)$ & $24(2,8)$ & $145(2,1 \%)$ & $\mathrm{p}=0,124$ \\
Dificuldades de enxergar & $95(1,6)$ & $40(4,7)$ & $135(2 \%)$ & $\mathrm{p}<0,001$ \\
Dificuldade com transporte coletivo ou privado & $107(1,8)$ & $26(3)$ & $133(1,9 \%)$ & $\mathrm{p}=0,011$ \\
Dificuldade de comunicação (ouvir ou falar) & $43(0,7)$ & $11(1,3)$ & $54(0,8 \%)$ & $\mathrm{p}=0,074$ \\
Medo de se perder & $23(0,4)$ & $15(1,8)$ & $38(0,5 \%)$ & $\mathrm{p}<0,001$ \\
Outra dificuldade & $20(0,3)$ & $5(0,6)$ & $24(0,4 \%)$ & $\mathrm{p}<0,001$ \\
Não observa nenhuma dificuldade & $4.053(66,9)$ & $423(49,4)$ & $4.476(64,7 \%)$ & $\mathrm{p}=0,247$
\end{tabular}

Tabela 3. Percepção dos locais públicos frequentados.

\begin{tabular}{lcrrr}
\hline & Idosos Jovens & Idosos Longevos & & \\
& $\mathbf{N}(\%)$ & $\mathbf{N}(\%)$ & Total & Valor p \\
\hline Inseguros & $1.590(26,3)$ & $215(25,1)$ & $1.805(26,1 \%)$ & $\mathrm{p}=0,466$ \\
Poucos bancos ou em mau estado & $1.189(19,6)$ & $138(16,1)$ & $1.327(19,2 \%)$ & $\mathrm{p}=0,014$ \\
Sujos (sem limpeza) & $912(15,1)$ & $112(13,1)$ & $1.024(14,8 \%)$ & $\mathrm{p}=0,124$ \\
Pouco iluminados & $542(8,9)$ & $47(8,0)$ & $589(8,5 \%)$ & $\mathrm{p}<0,001$ \\
Pouco arborizados & $486(8,0)$ & $41(7,8)$ & $527(7,6 \%)$ & $\mathrm{p}<0,001$ \\
Muitos degraus & $143(2,4)$ & $21(2,5)$ & $164(2,4 \%)$ & $\mathrm{p}=0,872$ \\
Não observa nenhum desses problemas & $1.873(30,9)$ & $224(26,1)$ & $2.097(30,3 \%)$ & $\mathrm{p}=0,004$ \\
Não sabe responder (não frequenta) & $1.215(20,1)$ & $293(34,2)$ & $1.508(21,8 \%)$ & $\mathrm{p}<0,001$
\end{tabular}

Tabela 4. Principais barreiras apontadas para atravessar as vias públicas.

\begin{tabular}{|c|c|c|c|c|}
\hline & $\begin{array}{c}\text { Idosos Jovens } \\
\qquad \mathrm{N}(\%)\end{array}$ & $\begin{array}{c}\text { Idosos Longevos } \\
\mathrm{N}(\%)\end{array}$ & Total & Valor p \\
\hline Os carros não param na faixa de pedestre & $1.181(19,5)$ & $149(17,4)$ & $1.330(19,2 \%)$ & $\mathrm{p}=0,141$ \\
\hline Falta faixas de segurança & $1.094(18,1)$ & $131(15,3)$ & $1.225(17,7 \%)$ & $\mathrm{p}=0,046$ \\
\hline Ambientes inseguros & $1.051(17,4)$ & $172(20,1)$ & $1.223(17,7 \%)$ & $\mathrm{p}=0,051$ \\
\hline O tempo do sinal muito curto para pedestres & $1.059(17,5)$ & $126(14,7)$ & $1.185(17,1 \%)$ & $\mathrm{p}=0,042$ \\
\hline Calçadas estreitas, mal conservadas ou inexistentes & $881(14,5)$ & $141(16,5)$ & $1.022(14,8 \%)$ & $\mathrm{p}=0,141$ \\
\hline Degraus (meio fio ou calçada) muito altos ou íngremes & $686(11,3)$ & $130(15,2)$ & $816(11,8 \%)$ & $\mathrm{p}=0,001$ \\
\hline Não existe sinaleira (semáforo) para pedestres & $477(7,9)$ & $62(7,2)$ & $539(7,8 \%)$ & $\mathrm{p}=0,511$ \\
\hline Ruas e ambientes mal iluminados & $270(4,5)$ & $43(5,0)$ & $313(4,5 \%)$ & $\mathrm{p}=0,461$ \\
\hline Calçadas com muito vendedores e carros estacionados & $205(3,4)$ & $24(2,8)$ & $229(3,3 \%)$ & $\mathrm{p}=0,370$ \\
\hline Ciclistas não respeitam os pedestres & $115(1,9)$ & $25(2,9)$ & $140(2 \%)$ & $\mathrm{p}=0,047$ \\
\hline Outro problema & $25(0,4)$ & $8(0,9)$ & $33(0,5 \%)$ & $\mathrm{p}=0,038$ \\
\hline Não apresenta dificuldade (exclui as outras) & $2.081(34,4)$ & $194(22,6)$ & $2.275(32,9 \%)$ & $\mathrm{p}<0,001$ \\
\hline Não sai de casa ou não anda pelas ruas (só usa carro) & $564(9,3)$ & $205(23,9)$ & $769(11,1 \%)$ & $\mathrm{p}<0,001$ \\
\hline
\end{tabular}


A segunda característica significativamente mais percebida, tendo seu percentual maior entre o grupo mais jovem $(18,1 \%)$ foi a falta das faixas de segurança. Os ambientes inseguros foram significativamente mais identificados pelos idosos longevos, correspondendo a 172 indivíduos. O tempo do sinal muito curto para pedestres foi significativamente mais enfatizado pelos idosos jovens $(17,5 \%)$. Não houve diferença estatística entre os idosos jovens e longevos quanto à percepção das calçadas estreitas, mal conservadas ou inexistentes, sendo estas referidas por 1022 indivíduos. Os degraus (meio fio ou calçada) muito altos ou íngremes foram significativamente mais relatados pelos longevos (15,2\%). A alternativa "não sai de casa ou não anda pelas ruas, só usa carro”, foi identificada por 769 (11,1\%) indivíduos, sendo estatisticamente significativa a maior prevalência entre o grupo mais velho (Tabela 4).

Em relação à percepção dos banheiros públicos são relatados o mau cheiro e a sujeira, sendo significativamente mais citados entre os idosos jovens, o que sugere que estes percebem mais essa característica porque saem mais de casa, conforme Tabela 5. A alternativa falta papel higiênico e/ou toalha também foi significativamente mais percebida pelos idosos jovens, com um total de 1212 dos entrevistados. Quanto à percepção dos "banheiros escassos ou não sabe onde tem" esta foi apontada por $788(13,0 \%)$ idosos jovens e 121 $(14,1 \%)$ longevos, não havendo diferença significativa entre os dois grupos. Enfatiza-se novamente o grande número total de idosos longevos que responderam significativamente a alternativa "não sai de casa, não sabe informar", correspondendo a 182 entrevistados.

\section{Discussão}

No Censo de 2010 observou-se que 13,8\% dos idosos tinham 80 anos ou mais, desta forma, a porcentagem de longevos entrevistados está bastante próxima do percentual do Censo, sendo esta uma amostra com representatividade populacional. Quanto ao gênero, esta pesquisa evidenciou que o percentual de mulheres foi maior que o dos homens. Fato também demonstrado em outros estudos onde as amostras são compostas na maioria por mulheres ${ }^{11-14}$. Na literatura internacional é relatado que os homens têm maior mortalidade quando comparados com as mulheres ${ }^{15}$. Esse fato é relacionado por alguns autores com o tabagismo, sendo esse hábito mais evidenciado no sexo masculino, gerando maior probabilidade de mortalidade por doenças respiratórias nesse grupo $^{16}$. Os homens também são mais acometidos por doença pulmonar obstrutiva crônica, insuficiência cardíaca e todas as formas de doenças arteriais (doenças com maiores índices de mortalidade), sendo as mulheres mais acometidas por comorbidades como osteoporose e depressão. Demonstrando, portanto, alguns motivos destas serem a maioria na população idosa ${ }^{17,18}$.

Neste estudo, sobre a frequência que os idosos saem de casa semanalmente, o número de longevos que referiram não ter esse hábito mostrou-se significativamente maior. Um estudo realizado na Cidade de Palmeira das Missões (RS), para avaliar a qualidade de vida e o nível cognitivo de idosos participantes de quatro grupos da terceira idade que se reuniam semanalmente, onde realizavam atividades como oficinas de artesanato, dança, passeios, exercícios físicos e socialização, identificou que a porcentagem de participação de idosos mais velhos foi inferior, predominando os idosos entre 60 e 69 anos e 70 e 79 anos $^{19}$. Estudos

Tabela 5. Percepção dos banheiros públicos.

\begin{tabular}{lrrrr}
\hline & Idosos Jovens & Idosos Longevos & & \\
& N (\%) & N (\%) & Total & Valor p \\
\hline Apresentam mau cheiro ou são sujos & $1.287(21,3)$ & $122(14,2)$ & $1.409(20,4 \%)$ & $\mathrm{p}<0,001$ \\
Falta papel higiênico e/ou toalha & $1.212(20)$ & $103(12)$ & $1.315(19 \%)$ & $\mathrm{p}<0,001$ \\
São escassos ou não sabe onde se tem & $788(13,0)$ & $121(14,1)$ & $909(13,1 \%)$ & $\mathrm{p}=0,369$ \\
São mal iluminados & $361(6,0)$ & $40(4,7)$ & $401(5,8 \%)$ & $\mathrm{p}=0,129$ \\
São de difícil acesso (sem corrimão, muitos degraus) & $119(2,0)$ & $14(1,6)$ & $133(1,9 \%)$ & $\mathrm{p}=0,508$ \\
Têm que ser pagos para usar (não são gratuitos) & $112(1,8)$ & $15(1,8)$ & $127(1,8 \%)$ & $\mathrm{p}=0,839$ \\
Não gosta de usar banheiro fora de casa & $1.360(22,5)$ & $170(19,8)$ & $1.530(22,1 \%)$ & $\mathrm{p}=0,083$ \\
Não observa nenhum desses problemas & $1.955(32,3)$ & $268(31,3)$ & $2.223(32,2 \%)$ & $\mathrm{p}=0,553$ \\
Não sai de casa, não sabe informar & $505(8,3)$ & $181(21,1)$ & $686(9,9 \%)$ & $\mathrm{p}<0,001$ \\
\hline
\end{tabular}


com esse mesmo tema abordam a importância das interações sociais para os idosos. É a partir do cotidiano de relações que os idosos tornamse conscientes de seus papéis como atores sociais, sendo capazes de contribuir para a sociedade ${ }^{20}$.

Em relação à falta de segurança para saírem de casa, esta pesquisa demonstrou que tanto os idosos jovens, quanto os longevos, percebem a falta de segurança como limitadora. A literatura demonstra a preocupação da população em relação à melhora da qualidade da segurança pública, o que possibilita os indivíduos de frequentarem parques abertos na comunidade de forma mais tranquila. Portanto, a percepção da falta de segurança pode influenciar diretamente o uso de áreas de lazer ${ }^{21}$.

A dificuldade de locomoção neste estudo foi maior no grupo longevo, sugere-se que isso ocorreu pelo fato desse grupo possuir mais morbidades e incapacidades. Um estudo realizado em Viçosa (MG) analisou a capacidade funcional de idosos longevos residentes em áreas urbanas do município, identificando que a perda da capacidade funcional obteve associação significativa com o fator "não visitar parentes e/ou amigos uma vez por semana", como também "não participar de alguma obra social ou não participar de eventos sociais". O mesmo estudo também demonstra que as chances de pessoas com mais de 85 anos apresentarem dependência funcional é três vezes maior do que em pessoas com menos de 85 anos $^{22}$. Estudos internacionais chamam atenção para a possível associação das perdas funcionais e a maior chance de dependência com o aumento da idade ${ }^{23-26}$. Os dados aqui relatados corroboram com a necessidade de identificação precoce, dos motivos que levam os idosos longevos a possuírem maior dificuldade de locomoção, para que assim, ações de assistência e prevenção possam ser direcionadas a esse segmento populacional.

Observou-se neste estudo que os idosos longevos são mais afetados pela falta de motivação para saírem de casa. Pode-se inferir que estes sejam mais acometidos por fatores que fazem com que os mesmos optem por ficar em suas residências, como por exemplo, a depressão. Um estudo que corrobora com o presente achado, avaliou em Pelotas (RS) a frequência de alguns sintomas depressivos na comunidade urbana de idosos do município. Foi demonstrado que as médias de sintomas depressivos (dentre eles, preferir ficar em casa e a falta de disposição) são mais frequentes em mulheres, com idades mais avançadas, e naqueles com baixa escolaridade ${ }^{27}$.
A falta de ter com quem sair prevaleceu no grupo dos longevos no presente estudo. Em estudo que analisou duas comunidades de longevos em regiões diferentes do país, uma em Ribeirão Preto (SP) e outra em Caxias do Sul (RS), demonstrou que em ambas as cidades existe um grande número de idosos que moram sozinhos, sendo essa proporção maior entre os gaúchos ${ }^{28}$. Fato este que corrobora com a hipótese de que muitos idosos longevos não saem de suas residências por não terem companhia e até mesmo apoio social adequado. No Guia Global Cidade Amiga do Idoso, a necessidade de reforço da rede de suporte comunitário aos idosos é enfatizada. Um estudo realizado em Porto Alegre (RS) para conhecer o perfil de idosos de uma determinada comunidade, destacou que quando questionados quanto ao apoio social informal, metade da amostra não se percebeu recebendo esse apoio ${ }^{29}$.

As dificuldades de enxergar e com transporte coletivo ou privado, mais citadas entre os idosos longevos, também foram evidenciadas nesta pesquisa como fatores contribuintes para que estes saíssem menos de suas residências. O grupo também relatou que o medo de se perder está entre esses fatores. Sugere-se que o medo de se perder esteja associado a déficits relacionados à memória dos idosos mais velhos. Estudos indicam que transtornos cognitivos, dentre estes, os problemas de memória, são mais frequentes conforme o aumento da idade e o envelhecimento ${ }^{30,31}$.

A percepção de poucos bancos ou em mau estado foi referida, em sua maioria, pelos idosos jovens. Idosos de todo o mundo relataram no Guia Global Cidade Amiga do Idoso que sentem dificuldade de andar pela cidade quando esta oferece poucos lugares para sentar e descansar. Estudos evidenciam a diminuição da capacidade funcional em idosos no Brasil no decorrer dos anos ${ }^{32-34}$. A perda gradativa da capacidade funcional pode ser responsável pela necessidade mais frequente dos idosos em sentar, de forma que a falta de banco ou bancos em estado precário podem impedir que o idoso usufrua dos atrativos que o meio urbano oferece.

Os ambientes públicos pouco iluminados foram mais percebidos nesta pesquisa como uma dificuldade para sair de casa pelos idosos jovens. Um estudo realizado em Florianópolis (SC) para expressar as características das condições de vida e saúde da população idosa demonstrou que um terço da amostra considerava sua visão ruim ou péssima. Entre os que diziam estar com alguma deficiência visual, a maioria referiu que este problema atrapalha suas rotinas bem como a realiza- 
ção de algumas atividades desejadas ${ }^{35}$. Sugere-se que a diminuição da acuidade visual somada à má iluminação de alguns locais públicos, podem ser fatores que afetam diretamente a escolha destes por não circularem à noite em ambientes da cidade, tendo assim, menos oportunidades de socialização e deslocamento frente às suas diferentes capacidades.

Observou-se no presente estudo um percentual maior de idosos mais velhos que referiram não saber responder sobre sua percepção dos locais públicos, porque não frequentavam os mesmos. Isso pode refletir a maior exclusão social deste segmento populacional. Enfatiza-se a necessidade de ações concretas a partir das políticas públicas do idoso que garantam a maior participação social desses indivíduos, tornando a cidade mais atrativa e acolhedora. O Estatuto do Idoso afirma que a proteção do envelhecimento é um direito social e que é dever do Estado assegurar ao idoso sua participação na vida comunitária ${ }^{36}$.

Neste estudo foi expressivo o número total de idosos que referiram à falta das faixas de segurança e o tempo de sinal muito curto para pedestres como barreiras para atravessarem as vias públicas, particularmente, no segmento mais jovem. Em um estudo realizado na Cidade de Barreto (SP), a existência da faixa de segurança para travessia de pedestres nas esquinas, foi identificada como a característica das calçadas e dos passeios públicos mais importante para a segurança de indivíduos idosos, segundo a percepção dos $\operatorname{mesmos}^{37}$. As alterações fisiológicas do envelhecimento, tais como a dificuldade de locomoção e o aumento do tempo de reação, somadas ao meio urbano mal estruturado e desorganizado, podem levar a um cansaço desencorajante para os idosos pedestres usufruírem dos lugares públicos ${ }^{38}$.

Os degraus (meio fio ou calçada) muito altos ou íngremes foram mais percebidos pelos idosos longevos deste estudo. É dever do Estado assegurar que algumas barreiras arquitetônicas e urbanísticas sejam eliminadas, para melhor circulação da população nos espaços da cidade. O Artigo $3^{\circ}$ da Lei 10.098/2000, afirma que o planejamento e a urbanização das vias públicas, dos parques e demais espaços comunitários, deverão ser elaborados de forma que proporcionem acessibilidade para pessoas com mobilidade reduzida, como no caso dos idosos longevos ${ }^{39}$.

Os idosos jovens deste estudo perceberam mais o mau cheiro dos banheiros públicos, possivelmente porque saem mais de casa e utilizam mais esses espaços. É referenciada na literatura a diminuição do olfato com o processo de envelhe- cimento $^{40}$. Mesmo podendo ter essa característica típica do envelhecimento o mau cheiro é referido, o que direciona para a realidade precária da higienização de alguns banheiros públicos, afetando diretamente o uso destes pelos idosos.

A falta de papel higiênico e/ou toalha nos banheiros públicos também foi uma característica enfatizada pelos idosos jovens da presente pesquisa. Estudos demonstram que a população idosa é acometida por distúrbios como a incontinência urinária, o que pode gerar a urgência dos idosos para utilização de um banheiro público $^{41,42}$. Evidencia-se a importância da melhora dos aspectos estruturais destes banheiros de forma que a qualidade de vida da população idosa frequentadora de ambientes comunitários não seja afetada. Países europeus tem direcionado a atenção para esses quesitos ao disponibilizar banheiros públicos de fácil acessibilidade e inclusive com dispositivos autolimpantes ${ }^{43}$.

\section{Considerações Finais}

Este estudo demonstrou a diferença de percepção que os idosos jovens e longevos gaúchos possuem do ambiente urbano em que vivem. A percepção de algumas dificuldades como poucos bancos, locais pouco iluminados, falta de faixas de segurança, tempo de sinal muito curto para pedestres, degraus muito altos e mau cheiro dos banheiros públicos foi maior entre os idosos jovens. Ao mesmo tempo em que os idosos longevos perceberam menos esses fatores, o grupo referiu que frequentava menos os ambientes comunitários, estabelecendo, assim, uma diminuição da relação desse contingente populacional longevo com suas cidades. Este tipo de achado apresenta-se como um dos principais fatores determinantes da qualidade de vida dos idosos longevos, sinalizando a necessidade de serem criadas estratégias que possibilitem ampliar a capacidade desses indivíduos sentiremse mais felizes e saudáveis no meio onde vivem.

A execução de ações formuladas a partir dos princípios da solidariedade, democracia e participação emergem como prioridades no combate às dificuldades apresentadas pelos idosos que participaram da pesquisa, as quais vêem influenciando negativamente no seu estilo de vida. Como alternativa, por exemplo, da falta de iluminação pública consideramos a importância do Estado se comprometer com a execução de obras que melhorem a iluminação nos espaços de uso comum das cidades do Rio Grande do Sul, tornando estes locais mais atrativos e seguros. 
Poderão ser elaboradas, em parceria com os governos e a sociedade, estratégias para a utilização e melhoria dos recursos existentes nas comunidades como praças, parques, espaços de convivência, dentre outros. Viabilizar a utilização desses espaços poderá promover bem estar e qualidade de vida da pessoa idosa. Em relação às demais dificuldades apresentadas nesta pesquisa, deve-se pensar em um contingente maior de investimentos para a construção de mais bancos e para melhorar a infraestrutura dos banheiros públicos.

A travessia de vias públicas apresenta-se neste estudo como uma das limitações encontradas pelos idosos jovens, justificada pela falta de faixas de segurança. Nesse sentido destacamos a relevância da construção de mais faixas de segurança e a realização de ações voltadas para a educação no trânsito, com foco na diminuição dos acidentes que podem acometer esses idosos que circulam pelos espaços urbanos.

Ações concretas a partir das políticas públicas já existentes, que possibilitem uma coexistência amigável entre o idoso e o ambiente urbano, são de suma importância para uma vida urbana satisfatória. Em especial, enfatiza-se a necessidade de mais estratégias para o cuidado do grupo longevo, para que suas interações cotidianas sejam ampliadas e assim seja garantido o seu envelhecimento ativo. Novos estudos com essa problemática podem servir como alicerce para a construção de um Brasil urbanamente mais acolhedor de seus idosos longevos.

\section{Colaboradores}

JHN Navarro, FP Andrade, TS Paiva, DO Silva, CF Gessinger e AJG Bós participaram igualmente de todas as etapas de elaboração do artigo. 


\section{Referências}

1. United Nations (UN). Department of Economic and Social Affairs, Population Division. World Urbanization Prospect: The 2011 Revision, Urban Population. [cited 2013 Jun 9]. Available from: http://esa.un.org/ unpd/wup/CD-ROM/Urban-Rural-Population.htm

2. United Nations (UN). Department of Economic and Social Affairs, Population Division. World Urbanization Prospect: The 2011 Revision, Rural Population. [cited 2013 Jun 9]. Available from: http://esa.un.org/unpd/ wup/CD-ROM/Urban-Rural-Population.htm

3. Instituto Brasileiro de Geografia e Estatística (IBGE). Séries Estatísticas e Séries Históricas, Revisão 2008. Projeção da População, Grupos Especiais de Idade, Idosos. [acessado 2013 jun 9]. Disponível em: http://seriesestatisticas.ibge.gov.br/series.aspx?vcodigo $=$ POP305\&s$\mathrm{v}=35 \& \mathrm{t}=$ revisao-2008-projecao-da-populacao-grupos -especiais-de-idade

4. United Nations Human Settlements. Living Conditions of Low-income Older Persons in Human Settlements, 2006. A Global Survey in Connection With The International Year of Older People. [cited 2013 Jun 9]. Available from: http://www.chs.ubc.ca/archives/?q=node/ 1050

5. Organização Mundial da Saúde (OMS). Guia Global: cidade amiga do idoso. Genebra: Publicações da OMS; 2008.

6. Organização Mundial da Saúde (OPAS). Envelhecimento Ativo: uma política de saúde. Brasília: Organização Pan-Americana de saúde; 2005.

7. Lunaro A, Ferreira MAG. Os Espaços Públicos e a Questão da Acessibilidade Sob o Ponto de Vista dos Idosos. Revista Ciência \& Engenharia 2005; 15(2):67-72.

8. Marafon LP, Cruz IBM, Schwanke CHA, Moriguchi EH. Preditores Cardiovasculares da Mortalidade em Idosos Longevos. Cad Saude Publica 2003; 19(3):799808.

9. Cruz IBM, Almeida MSC, Schwanke CHA, Moriguchi EH. Prevalência de Obesidade em Idosos Longevos e sua Associação com Fatores de Risco e Morbidades Cardiovasculares. Rev Assoc Med Bras 2004; 50(2):172177

10. Instituto Brasileiro de Geografia e Estatística (IBGE). Censo Demográfico 2010: pessoas de 5 anos ou mais de idade, alfabetizadas, por grupos de idade, segundo as Grandes Regiões e as Unidades da Federação. [acessado 2013 jun 9]. Disponível em: http://www.censo2010. ibge.gov.br/sinopse/index.php?dados $=12$

11. Martikainen P, Aromaa A, Heliovaara M, Klaukka T, Knekt P, Maatela J, Lahelma E. Reliability of perceived health by sex and age. Soc Sci Med 1998; 48(8):11171122.

12. Siqueira FV, Facchini LA, Piccini RX, Tomasi E, Thumé E, Silveira DS, Vieira V, Hallal PC. Prevalência de quedas em Idosos e Fatores Associados. Rev Saude Publica 2007; 41(5):749-756.

13. Mastroeni MF, Erzinger ZS, Mastroeni SSBS, Silva NN, Marucci MFN. Perfil Demográfico de Idosos da Cidade de Joinville, Santa Catarina: Estudo de base domiciliar. Rev Bras Epidemiol 2007; 10(2):190-201.

14. Braga C, Lautert L. Caracterização sociodemográfica dos idosos de uma comunidade de Porto Alegre, Brasil. Rev Gaúcha Enferm 2004; 25(1):44-55.
15. Mackenbach JP, Kunst AE,Groenhof F, Borgan JK, Costa G, Faggiano F, Józan P, Leinsalu M, Martikainen P, Rychtarikova J, Valkonen T. Socioeconomic Inequalities in Mortality Among Women and Among Men: An International Study. Am J Public Health 1999; 89(12):1800-1806.

16. Prescott E, Osler M, Andersen PK, Hein HO, BorchJohnsen K, Lange P, Schnohr P, Vestbo J. Mortality in Women and Men in Relation to Smoking. Int J Epidemiol 1998; 27(1):27-32.

17. Kannegaard PN, Mark SVD, Eken P, Abrahamsen B. Excess Mortality in Men compared with Women Following a Hip Fracture. National Analysis of Comedications, comorbidity and Survival. Age Ageing 2010; 39(2):203-209.

18. Repetto L, Fratino L, Audisio RA, Venturino A, Gianni W, Vercelli M, Parodi S, Dal Lago D, Gioia F, Monfardini S, Aapro MS, Serraino D, Zagonel V. Comprehensive Geriatric Assessment Adds Information to Eastern Cooperative Oncology Group Performance Status in Elderly Cancer Patients: An Italian Group for Geriatric Oncology Study. J Clin Oncol 2002; 20(2):494-502.

19. Leite MT, Winck MT, Hildebrandt LM, Kirchner RM, Silva LAA. Qualidade de Vida e Nível Cognitivo de Pessoas Idosas Participantes de Grupos de Convivência. Rev Bras Geriatr Gerontol 2012; 15(3):481-492.

20. Araújo LF, Coutinho MPL, Carvalho VAML. Representações Sociais da Velhice Entre Idosos que Participam de Grupos de Convivência. Psicologia Ciência e Profissão 2005; 25(1):118-131.

21. Cohen DA, McKenzie TL, Sehgal A, Williamson S, Golinelli D, Lurie N. Contribution of Public Parks to Physical Activity. Am J Public Health 2007; 97(3):510-514.

22. Nogueira SL. Fatores determinantes da capacidade funcional em idosos longevos. Rev Bras Fisioter 2010; 14(4):322-329.

23. Murabito JM, Pencina MJ, Zhu L, Kelly-Hayes M, Shrader P, D'Agostino RB. Temporal trends in self-reported functional limitations and physical disability among the community-dwelling elderly population: the Framingham hearth study. J Public Health 2008; 98(7):1256-1262.

24. Ishizaki T, Kai I, Kobayashi Y, Matsuyama Y, Imanaka Y. The effect of aging on functional decline among older Japanese living in a community: a 5-year longitudinal data analysis. Aging Clin Exp Rev 2004; 16(3):233-239.

25. Femia EE, Zarit SH, Johansson B. The disablement process in very late life: a study of the oldest-old in Sweden. J Gerontol B Psychol Sci Soc Sci 2001; 56(1):12-23.

26. Barbosa AR, Souza JMP, Lebrão ML, Laurenti R, Marucci MFN. Functional limitations of Brazilian elderly by age and gender differences: data from SABE Survey. Cad Saude Publica 2005; 21(4):1177-1185.

27. Gazalle FK, Lima MS, Tavares BF, Hallal PC. Sintomas Depressivos e Fatores Associados em População Idosa no Sul do Brasil. Rev Saude Publica 2004; 38(3):365371.

28. Rosset I, Roriz-Cruz M, Santos JLF, Haas VJ, Fabrício -Wehbe SCC, Rodrigues RAP. Diferenciais Socioeconômicos e de Saúde entre Duas Comunidades de Idosos Longevos. Rev Saude Publica 2011; 45(2):391-400. 
29. Paskulin LMG, Vianna LAC. Perfil Sóciodemográfico e Condições de Saúde Autoreferidas de Idosos de Porto Alegre. Rev Saude Publica 2007; 41(5):757-768.

30. Lourenço RA, Veras RP. Mini-Exame do Estado Mental: características psicométricas em idosos ambulatoriais. Rev Saude Publica 2006; 40(4):712-719.

31. Laks J, Batista EMR, Guilherme ERL, Contino ALB, Faria MEV, Figueira I, Engelhardt E. O Mini Exame do Estado Mental em Idosos de Uma Comunidade. Arq Neuropsiquiatr 2003; 61(3):782-785.

32. Parahyba MI, Veras R, Melzer D. Incapacidade Funcional entre as mulheres idosas no Brasil. Rev Saude Publica 2005; 39(3):383-391.

33. Parahyba MI, Simões CCS. A Prevalência de Incapacidade Funcional em Idosos no Brasil. Cien Saude Colet 2006; 11(4):967-974

34. Aires M, Paskulin LMG, Morais EP. Capacidade Funcional de Idosos Mais Velhos: estudo comparativo em três regiões do Rio Grande do Sul. Rev Latino-Am Enfermagem 2010; 18(1):11-17.

35. Benedetti TB, Petroski EL, Gonçalves LT. Condições de Saúde nos Idosos de Florianópolis. Arquivos Catarinenses de Medicina 2006; 35(1):44-51.

36. Brasil. Lei N.o 10.741, de $1^{\circ}$ de outubro de 2003. Dispõe sobre o Estatuto do Idoso e dá outras providências. Diário Oficial da União 2003; 1 out.

37. Lunaro A. Avaliação dos Espaços Urbanos Segundo a Percepção das Pessoas Idosas - SP [dissertação]. São Carlos: Universidade de São Carlos; 2006.

38. Micheletto TMGP. O Risco do Idoso Pedestre nas Vias Urbanas. São Paulo: Companhia de Engenharia de Tráfego [Notas técnicas]; 2011.
39. Brasil. Lei N. ${ }^{\circ} 10.098$, de 19 de dezembro de 2000. Dispõe sobreos critérios básicos para a promoção da acessibilidade das pessoas portadoras de deficiência ou com mobilidade reduzida e dá outras providências. Diário Oficial da União 2000; $19 \mathrm{dez}$

40. Lima JP. A Influência das Alterações Sensoriais na Qualidade de Vida do Idoso. Rev Científica Eletrônica de Psicologia [periódico na internet]. 2007 maio [acessado 2013 jun 9]; (8). Disponível em: http://www.revista.inf. br/psicologia08/pages/artigos/edic08-anov-art03.pdf

41. Honório MO, Santos SMS. Incontinência Urinária e Envelhecimento: impacto no cotidiano e na qualidade de vida. Rev Bras Enferm 2009; 62(1):51-56.

42. Abreu NS, Baracho ES, Tirado MGA, Dias R. Qualidade de Vida na Perspectiva de Idosas com Incontinência Urinária. Rev Bras Fisioter 2007; 11(6):429-436.

43. O Guia de Paris. Como funciona o banheiro público em Paris [reportagem Blog]. 2011; janeiro. [acessado 2013 jun 9]. Disponível em: http://oguiadeparis.blogspot. com.br/2011/01/como-funciona-o-banheiro-publico -em.html

Artigo apresentado em 11/04/2014

Aprovado em 13/06/2014

Versão final apresentada em 15/06/2014 